\title{
Highly Parallel Computation
}

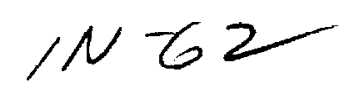

\author{
Peter J. Denning \\ Walter F. Tichy
}

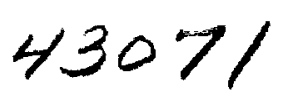

034

August 19, 1990

RIACS Technical Report TR-90.35

NASA Cooperative Agreement Number NCC2-387

(NASA-CR-1B8371) HIGHLY PARALLEL

N92-11698

COMPUTATION (ResearCh Inst. For Advanced

Computer Science) $34 \mathrm{P} \quad \mathrm{CSCL} 09 \mathrm{~B}$ 


\section{$=$}

. 


\title{
Highly Parallel Computation
}

\author{
Peter J. Denning \\ Walter F. Tichy \\ Research Institute for Advanced Computer Science \\ NASA Ames Research Center
}

RIACS Technical Report TR-90.35

August 19, 1990

Highly parallel computing architectures are the only means to achieve the computational rates demanded by advanced scientific problems. A decade of research has demonstrated the feasibility of such machines and current research focuses on which architectures are best suited for particular classes of problems. The architectures designated as MIMD and SIMD have produced the best results to date; neither shows a decisive advantage for most near-homogeneous scientific problems. For scientific problems with many dissimilar parts, more speculative architectures such as neural networks or dataflow may be needed.

This is a preprint of an article to appear in Science magazine.

Work reported herein was supported in part by Cooperative Agreement NCC2-387

between the National Aeronautics and Space Administration (NASA) and the Universities Space Research Association (USRA). 


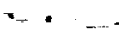




\title{
Highly Parallel Computation
}

\author{
Peter J. Denning and Walter F. Tichy
}

August 19, 1990

This paper has been accepted for publication

in Science magazine during fall 1990.

P. J. Denning is Research Fellow of the

Research Institute for Advanced Computer Science

NASA Ames Research Center

Moffett Field, CA 94035 USA

W. F. Tichy is in the Computer Science Department of the

University of Karlsruhe, FRG

\begin{abstract}
Highly parallel computing architectures are the only means to achieve the computational rates demanded by advanced scientific problems. A decade of research has demonstrated the feasibility of such machines and current research focuses on which architectures are best suited for particular classes of problems. The architectures designated as MIMD and SIMD have produced the best results to date; neither shows a decisive advantage for most near-homogeneous scientific problems. For scientific problems with many dissimilar parts, more speculative architectures such as neural networks or dataflow may be needed.
\end{abstract}



Computation has emerged as an important new method in science. It gives access to solutions of fundamental problems where pure analysis and pure experiment cannot reach. Aerospace engineers, for example, estimate that a complete numerical simulation of an aircraft in flight could be performed in a matter of hours on a supercomputer capable of sustaining at least 1 trillion floating point operations per second (teraflops, or tflops). Researchers in materials analysis, oil exploration, circuit design, visual recognition, high energy physics, cosmology, earthquake prediction, atmospherics, oceanography, and other disciplines report that breakthroughs are likely with machines that can compute at a tflops rate.

The fastest workstations today operate at maximum speeds of slightly beyond 10 million flops (megaflops, or mflops). In contrast, the fastest supercomputers have peak rates in excess of 1 billion flops (gigaflops, or gflops) -- e.g., the NEC SX-2 is rated at 1.0 gflops and Cray Y-MP at 2.7 gflops. Even faster ones are coming: the 4-processor NEC SX-3 (1990) will have peak rate of 22 gflops and the Cray 4 (1992) 128 gflops. When recompiled for these machines, standard Fortran programs typically realize $10 \%$ to $20 \%$ of the peak rate. When algorithms are carefully redesigned for the machine architecture, they realize $70 \%$ to $90 \%$ of the peak rate [1]. There is an obvious payoff in learning systematic ways to design algorithms for parallel machines.

Gordon Bell anticipates that machines capable of 1 tflops containing thousands (or even millions) of processors will be available as early as 1995 [2]. For example, IBM Research is developing the Vulcan machine, which will consist of $32,768\left(2^{15}\right) 50$-mflops processors, and Thinking Machines Corporation is considering a Connection Machine with over a million $\left(2^{20}\right)$ processors. These supermachines may cost on the order of $\$ 50$ 
millions apiece. Bell anticipates that low-cost, single-processor, reduced instruction set chips (RISC) with speeds on the order of 20 mflops will be common in workstations by 1995. It is clear that tflops machines will be multicomputers consisting of large numbers of processing elements (processor plus memory) connected by a high-speed message exchange network. Smaller multicomputers will proliferate in the next five years: we must learn to program them.

Speedup is a common measure of the performance gain from a parallel processor. It is defined as the ratio of the time required to complete the job with one processor to the time required to complete the job with $N$ processors [3]. Perfect speedup, a factor of $N$, can be attained in one of two ways. In a machine were each piece of the work is permanently assigned to its own processor, it is attained only when the pieces are computationally equal and processors experience no significant delays in exchanging information. In a machine where work can be dynamically assigned to available processors, it is attained as long as the number of pieces of work ready for processing is at least $N$.

In discussing speedup, it is important to distinguish between problem size and computational work. Problem size measures the number of elements in the data space and computational work the number of operations required to complete the solution. For example, an $N \times N$ square matrix occupies $N^{2}$ storage locations and it takes about $N^{3}$ operations to form the product of two of them. Doubling the dimension multiplies the storage requirement by four and the computational work by eight. Conversely, doubling the number of processors would permit multiplying two matrices of dimension $26 \%$ larger in the same amount of time. This has important consequences for multiprocessors: 
there may be too few processors available to achieve speedup that is linear in problem size. The best we can achieve is speedup that is linear in the number of processors.

A study in 1988 at Sandia National Laboratory provided the first case of nearperfect speedup for three problems involving the solutions of differential equations on a machine with 1024 processors [4]. Later that year, Geoffrey Fox published a study of 84 parallel algorithms reported in the scientific literature and concluded that $90 \%$ of them could be extrapolated to larger machines with speedups proportional to the number of processors [5]. These results give considerable grounds for optimism about speedup on other problems.

The central question of the early 1980 s was whether parallel computation would become practical. This question has been settled and we have moved on to bigger questions. What are the best parallel architectures for given classes of problems? How can we partition a given problem into thousands of parts that can be independently executed on different processors? How do we design algorithms so that delays of interprocessor communication can be kept to a small fraction of the computation time? How can we design the parts so that the load can be distributed evenly over the available processors? How can we design the algorithms so that the number of processors is a parameter and the algorithm can be configured dynamically for the available machine? How can we prove that a parallel algorithm on a given machine meets its specifications? How do we debug programs, especially when the results of flawed parallel algorithms may not be precisely reproducible? 


\section{Multiprocessor Architectures}

It is useful to review the basic approaches in the organization of multiprocessor computers suitable for parallel computation. These computers are classified in three major dimensions:

i. Shared memory versus distributed memory. In a shared memory computer each processor has access to all the computational memory; in a distributed memory computer, each has access only to its local memory and must exchange messages with other processors to obtain nonlocal data.

ii. Coarse grain versus fine grain. The unit of computational work allocated to a processor is called a grain. In coarse mode, a grain contains many data elements and, in fine mode, a grain contains one (or very few) data elements.

iii. SIMD versus MIMD. In one form of multiprocessor, a control processor broadcasts instructions one at a time to all the other processors, and each of them applies the current instruction to the data in its local memory. This mode is called SIMD for "single instruction stream, multiple data streams." When each of the processors is allowed to execute its own, separate program, the mode is called MMMD for "multiple instruction stream, multiple data streams." These two designations are part of a larger taxonomy proposed by Michael Flynn in 1972 [6].

In the following subsections we comment on these distinctions. Figure 1 shows four practical architectures exemplifying them. 


\section{Interconnection Network}

An important component of all multiprocessor architectures is the interconnection network. It provides paths by which processors can make requests to read, write, or lock memory locations, or can exchange messages with other processors. The interconnection network of a machine with thousands of processors should satisfy four properties:

i. Full connectivity. Any processor should be able to send a message to any other processor. (Connectivity from virtual addressing is possible but untested in parallel machines.)

ii. Parallel message exchange. The network should be capable of handling requests from all processors simultaneously, with minimal delays from contention at switch points. There should be no singular point in the network, such as the root of a tree or the hub of a star, that is a bottleneck.

iii. Short diameter. The longest path should be of lower order than the number of processors; order of $\log N$ for $N$ processors is acceptable.

iv. Scalable. The number of wires and switch points in the network should be of lower order than the square of the number of processors; order of $N \log N$ for $N$ processors is acceptable.

Many interconnection networks satisfy these properties [7]. One of the simplest is the hypercube, which connects each of the $N=2^{k}$ processors to $k$ others, has diameter $k$, and has wire and switch-point growth proportional to $N \log N[8]$.

Some multiprocessor computers use interconnection networks that do not satisify these properties but are cost effective because the number of processors is small. One is the shared bus, which can be used by only one processor at a time; it fails on Property (ii) 
because when the number of processors approaches 100 in current designs, bus contention becomes so severe that the bus saturates and limits the speed of the machine. Examples of computers that use it are Sequent Symmetry and Encore Multimax. Another is the crossbar switch, which provides every processor with a path to every memory; it fails on Property (iv) because it contains $N^{2}$ switch points and becomes unwieldy for more than a few hundred processors. Examples of computers that use it are Cray X-MP and Cray Y-MP, where the crossbar switching logic is distributed among the individual memory units.

\section{Shared v. Distributed Memory}

The shared memory architecture was introduced by Burroughs in the B5000 machine in the late 1950s and is used today in machines like the Sequent Balance and Encore Multimax. It gives all processors equal access to all memories through the interconnection network. Messages of any length can be exchanged in the fixed time required to exchange the address of the message header. The distributed memory architecture gives each processor direct access to only one memory unit, the local memory; access to other data is gained by sending messages through the interconnection network. Message exchange time is proportional to message length. This approach is economical if local accesses are more frequent than other accesses.

The strategy of designing algorithms for full sharing of all memory by all processors has an important fundamental limitation. Because individual memory modules can be accessed by only one processor at a time, all but one of the processors seeking access to a given module will be blocked for the duration of that module's cycle 
time. Baskett and Smith showed that when $N$ processors share access to $M$ memories, the fraction of processors blocked during each memory cycle is approximately $[9,10]$

$$
\sqrt{1+(N / M)^{2}}-N / M
$$

For $N=M$, about $40 \%$ of the processors will be blocked. The designers of the BBN Corporation's Monarch machine (65,536 processors and memories, 1 microsecond memory cycle time) say they can reduce this number to as little as $10 \%$ by having two ports into every memory [11].

The critical issue is the memory address pattern generated by each processor. If most of a given processor's references are concentrated in a small region of the address space, that region can be stored in a fast local memory attached to the processor; blocking will be negligible because interference will be limited to the few other processors whose favored regions overlap [10]. Because most parallel algorithms can be designed to localize the reference patterns of each processor, the distributed memory architecture will continue to be favored by designers.

\section{Coarse v. Fine Grains}

In analyzing parallel algorithms, we must distinguish two disjoint ways a processor can spend its time. One is computation, the time spent performing instructions. The other is communication, the time spent sending, receiving, or waiting for messages from other processors; communication time may vary according to path length to the processor holding the data and it may take 1 to 1,000 instruction times or more per message. Although all algorithms have communication time for input and output $(\mathrm{I} / \mathrm{O})$, the commúnication time required to synchronize the parts of a parallel algorithm is a cost 
that is not present in sequential algorithms.

The computational utilization $U_{i}$ of a processor $i$ is the fraction of time that processor is executing computational instructions; thus $1-U_{i}$ is the fraction of time that processor is executing communication instructions or waiting for messages. The speedup attained by a computation with $N$ processors is at most $U_{1}+U_{2}+\ldots+U_{N}$; it may be less if portions of the computation are repeated in several grains. The maximum speedup of $N$ will be achieved by an algorithm in which the computational utilization of each processor is near 1 and there is little redundant computation.

In many algorithms for physical problems, each processor is assigned a region of space containing a cluster of points on the grid over which the differential equations are solved. In a two-dimensional grid, a square with $k$ points on an edge will have computational work proportional to the number of points, $k^{2}$, and communication proportional to the perimeter, $4 k$. With $k$ sufficiently large, the computational work will be large compared to the communication, which means each $U_{i}$ will be close to 1 and $N$ such processors will produce nearly perfect speedup.

Now we see why grain size is important. The architecture will determine the cost of a communication step relative to a computation step. If the cost is high, the algorithm designer will favor large grains containing many instructions for each message; the number of subprograms will be a small function of the problem size. If the cost is low, the algorithm designer can afford small grains and the number of grains will be proportional to problem size. In specifying algorithms that will scale for larger machines, designers tend to choose grain sizes at the point of diminishing returns between computation and communication; for this reason, when given a machine with 
more processors, they use it for a larger problem at the same grain size rather than for the same problem with a smaller grain size [4].

The attraction of fine grains is that they afford the largest possible amount of speedup. They are practical in certain limited cases today, most often signal and image processing problems and problems involving particle tracing. Machines illustrating this are the Connection Machine [12] and the Goodyear/NASA Massively Parallel Processor (MPP) [13]. In these cases, the machines are able to move a data element between immediately neighboring processors in time comparable to the instruction time, and many computations over grids of such elements will achieve individual processor computational utilizations of 0.5 or greater at the finest grain.

\section{SIMD v. MIMD}

A fundamental question in the design of parallel algorithms is how to guarantee that, when a processor executes an instruction, the operands of that instruction have already been computed by previous instructions. Without this guarantee, the results of the comptuation can be indeterminate and depend on the relative speeds of the processors (race conditions). The mechanism that provides this guarantee is called synchronization.

Synchronization is straightforward in standard sequential single-processor machines, where instructions are executed one at a time. The results of each instruction are left in registers or in memory for access by later instructions. Optimizing compilers for such machines may exchange the order of instructions that do not provide data to each other. A direct extension of this mode for multiprocessing appears on machines of the SIMD type, where each instruction is simultaneously obeyed by all the processors. 
An example will illustrate. Suppose that a difference equation on a grid calls for averaging the values at the four nearest neighbors of a point; the programming language expression for the operation to be applied at point $(i, j)$ would read

$$
v(i, j)=[v(i-1, j)+v(i+1, j)+v(i, j-1)+v(i, j+1)] / 4
$$

On the SMMD machine, we can associate one data processor with each point on the grid; its memory holds the value $v(i, j)$. The control processor broadcasts the instructions implementing Expression (1) to all the data processors, which obey them using their own particular values of $i$ and $j$. Programs of this kind are easy to understand because they look almost the same as their counterparts for a single-processor machine. Hillis and Steele, in fact, say that the best way to think of SIMD programming is sequential programming in which each operation applies simultaneously to sets of data rather than to individual data elements [14]. It is impossible to program races in SIMD algorithms.

Under the MIMD mode, each processor has its own separate program of instructions to obey. The programs need not be identical. Now the machine must provide explicit means for synchronization. The hardware must supply buffers for passing messages between processors, flags to indicate the arrivals of signals and messages, and instructions that stop and wait for the flags. The programmer must use these synchronization instructions where a definite order of events must be established. For example, Expression (2) becomes

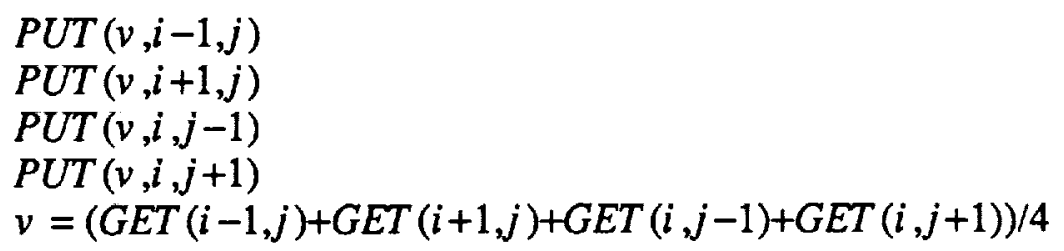


where $P U T$ sends a message containing the value of $v$ to a designated processor and $G E T$ waits until a message is received from a designated processor; a $G E T$ must match the corresponding PUT on the sending processor. Obviously this increases the programming effort and exposes the programmer to errors that arise when these new operations are used improperly. (Think what would happen if the four PUT statements did not all precede the GETs.)

The main limitation of the SIMD architecture is its restriction that all processors must execute the same instruction. Even in highly regular problems there are differences, such as the evaluation of boundary conditions, that require different algorithms for some processors than others. The machine must shut off boundary processors while broadcasting the instructions for interior nodes, and it must shut off interior processors while broadcasting instructions for boundary nodes. The need to shut off some of the processors lowers the utilization of the machine and the speedup it can attain. An MIMD architecture, which can execute the interior and boundary algorithms in parallel, does not suffer from this limitation.

\section{Practical Considerations}

There are at least eight distinguishable architectures corresponding to the various combinations of the factors above. In practice to date in scientific computing only three of these possibilities have been used:

MIMD coarse shared MIMD coarse distributed SIMD fine distributed
Sequent, Encore, Alliant, Convex, Cray hypercubes (Intel, Ametek, NCube)

Connection Machine, MPP 
There are two reasons for this. First, the shared memory architecture has been of limited use in large computations because fewer than a hundred processors are enough to saturate the common bus; such architectures do not extend to thousands of processors. Moreover, there are no reported test cases in which shared memory was a distinct advantage even when a small number of processors was sufficient [5]. Second, the grain size is normally the consequence of the communication structure of the machine and the nonlocal referencing patterns of the algorithm. MIMD machines to date have used coarse grains because synchronization costs would be too high with fine grains. Only the SIMD architecture has been successful with fine grains, and then only with each processor having its own local memory.

The predominence of these three architecture types today does not mean that others are forever impractical. We will discuss later the dataflow architecture, which is capable of supporting fine grain parallelism within the MMD mode and may become practical by the end of the decade.

As discussed by Hopfield and Tank, neural networks can be used for special purpose combinatorial optimization and pattern recognition problems [15]. They represent another architectural type that can be used for highly parallel computation. They are not of direct interest in the numerical computations that predominate in computational science, but they may be of indirect interest for ancillary combinatorial issues such as automatic grid generation and mapping grids to the nodes of a hypercube. 


\section{Problem Classes}

Let us turn now to the types of problems that have proved to be most amenable to efficient execution on highly parallel computers. The good news is that for a wide range of scientific problems, at least one of the three architectural types noted above works well [5].

Fox has proposed a classification of problems into three broad categories [5]. Synchronous problems are ones in which the physical equations specify the behavior at every point in the data space for every small increment of time. Loosely synchronous problems are ones for which there are embedded time sequences (renewal points) at which the physical equations specify the values of the data elements; in between these times there is no global specification of the data values in local regions of data space. Asynchronous problems are all the rest. Fox says that most of the results in the literature have been obtained for the first two types classes of problems and that we have not yet learned how to divide problems into dissimilar pieces that can keep an MIMD machine busy.

\section{Single-Function Problems}

We now reformulate these notions of problem classes in a way that more clearly reveals which types of problems are best suited for types of architectures.

Many computational problems have the characteristic that a simple procedure must be applied uniformly across a large number of data elements organized within a data structure. We can specify the procedure by a sequential algorithm in which each step is an operation applied simultaneously to all the data elements. The design of such "data 
parallel" algorithms closely resembles ordinary programming in languages such as Fortran or $\mathrm{C}$.

Physical problems modeled by a set of differential equations are common paradigms for data parallel algorithms. The continuum equations are modeled by a set of difference relations among dependent quantitites associated with points on a discrete grid. The difference relations are usually the same for all points except the boundaries. In a data parallel algorithm each grid point is assigned its own processor that contains a program to evaluate the difference relation. Because the difference relation depends only on the immediately adjacent grid points, each processor need communicate only with a small number of others in its neighborhood.

The class of problems amenable to data parallel solution is by no means limited to differential equation models. Others include:

i. Searching. Find data elements satisfying a given property. If processors are as numerous as data elements, the search can be completed in a constant amount of time independent of the data set and the result reported in an additional $\log N$ time.

ii. Sorting. Arrange a sequence of data elements in order. If processors are as numerous as data elements, the sort can be completed in time proportional to $\log ^{2} N$ on $N$ processors.

iii. Joining tables in a database. Form a new table from two others having a common column, by combining a record from one table with a record in the other whenever they have the same value in the common column. This can be done in time proportional to the size of the larger table if processors are numerous. 
iv. Computational Geometry. Find the convex hull of a set of points. With $N$ processors and $N$ points, this can be done in average time proportional to $\log ^{2} N$.

v. Solving Linear Equations. Find the solution of a set of equations of the matrix form $A x=b$. With $N$ processors and $N$ unknowns, the parallel Gauss-Jordan method obtains the solution in time proportional to $N^{2}$; with $N^{2}$ processors, the time drops to $N \log N$.

vi. Fast Fourier Transform. Find the one-dimensional Fourier transform of a series of points. With $N$ processors and $N$ points, this can be done in time proportional to $\log N$

This list is merely suggestive: it indicates that a large variety of subproblems that commonly arise in computational libraries are data parallel and subject to considerable speedup on machines containing large numbers of processors [16,17].

Practical data parallel algorithms must be designed to adapt to the number of processors actually available. For example, $1,000,000$ data elements can be searched by 1,000 processors in time proportional to $\log 1000$ by performing a binary search on the 1,000 elements allocated to each processor. Optimal combinations of sequential and parallel components are open problems in parallel algorithm design.

It is important to remember that data parallel algorithms cannot be universally guaranteed to keep all the processors busy all the time. An illustration is an image processing algorithm that operates in two passes. On the first, the algorithm determines local features of chunks of the image and, on the second, it locates contours by joining the local features across chunks. Processors assigned to chunks having few features will also have little work to do. Because the proportion of processors that can be kept busy is 
dependent on the input data, one cannot expect that the speedup will be proportional to the number of processors.

\section{Multiple-Function Problems}

Many computational problems involve many functions composed together. Examples include finite element analysis over nonhomogeneous rigid structures, multizone fluid flow calculations, circuit simulations, fluid flows in nonhomogeneous subterranean formations, and multidisciplinary models. These problems cannot be regarded as data parallel; they must instead be regarded as a network of machines performing different functions and exchanging data. They correspond to Fox's asynchronous problems. Their algorithms are today commonly written in C or Fortran. Many researchers believe that process-oriented languages such as Occam [18] and functional composition languages such as VAL [19] or FP [20] would produce more concise descriptions of algorithms for these problems.

\section{Architectural Matching}

From these descriptions it is obvious that single-function (data parallel) problems are well suited to the SIMD architecture, and multi-function problems are well suited to the MIMD architecture. A major impediment to solving multi-function problems to date has been the lack of programming languages that express functional composition easily. The main barrier to the widespread use of such languages is cultural. The scientific community has used Fortran for so many years that programming with new languages is unfamiliar and will remain untried as long as the scientific investigator sees no value to 
learning it. The ability to express solutions to multi-function problems may be a sufficient motivation to learn this later in the 1990s.

\section{Connection Machine}

To make concrete the previous points about solving single function problems on an SIMD machine, we will consider the architecture and programming of a particular SIMD machine, the Connection Machine 2 manufactured by Thinking Machines Corporation.

The Connection Machine model 2 (CM2) is an SIMD computer with $65,536\left(2^{16}\right)$ processors connected in a 16-dimensional hypercube network. Each processor has 32 kilobytes of local memory; the entire primary memory of a CM2 is 2 gigabytes $\left(2^{31}\right.$ bytes). The processors collectively have the capacity to be a supercomputer that solves very large problems with data parallel methods.

The CM2 cycles between intervals of instruction execution and message exchange. At the start of an instruction interval, the control processor broadcasts an instruction to all processors; the subset of them that are enabled execute that instruction using data in their local memories. During a message exchange interval, processors copy values required during the next instruction interval. It is easy for compilers to determine the source and destination addresses of these messages. If an algorithm uses many long paths in the network, the message interval can be 50-250 floating-point instruction times, severely limiting the computational rate of the machine.

The CM2 configures algorithms for the number of processors actually present through the method of virtual, or simulated, processors. The programmer designs an 
algorithm just once, assuming that the machine has the required number of processors. The compiler assigns sets of the programmer's virtual processors to each available processor on the CM2, and that processor simulates the execution of all the virtual processors assigned to it. The maximum number of virtual processors is limited by the available memory. As an example, we can assign a virtual processor to every point in a 1024 by 1024 image ( $2^{20}$ points); each of the CM2's $2^{16}$ processors must simulate 16 virtual processors, each limited to $1 / 16$ th of the memory and $1 / 16$ the speed of a processor.

Programmers use standard languages (Lisp, Fortran, C) on the CM2. We will review how standard control statements operate on the CM2. Consider a selection statementof the form IF C THEN A ELSE B:

i. The control processor broadcasts the instructions that evaluate the test $C$; at the end of this sequence, each processor contains the value TRUE or FALSE. The control processor broadcasts an instruction telling all processors containing FALSE to turn themselves off.

ii. The control processor broadcasts the instructions for the clause A; those instructions will be obeyed by the subset of processors still on. At the completion of this sequence, the control processor broadcasts an instruction telling all processors to reverse their status between on and off.

iii. The control processor broadcasts the instructions for the clause B; those instructions will be obeyed by the subset of processors now on. At the completion of this sequence, the control processor broadcasts an instruction telling all processors to turn themselves on. 
The CM2 implements the on and off status of processors with a one-bit register per processor called the context flag. When the context flag is FALSE, the associated data processor is off; when off, it obeys only instructions that unconditionally manipulate context flags.

An iteration statement such as WHILE C DO A works similarly. The control processor broadcasts the instructions of the test $\mathrm{C}$ and then the command for all processors containing FALSE to turn themselves off. It then broadcasts the instructions of A and only the processors still on execute it. This is repeated until all processors have shut themselves off. Thereafter the control processor instructs them all to turn themselves on again.

Because the programming syntax for the $\mathrm{CM} 2$ is basically unchanged from familiar sequential machines, many algorithms can easily be converted for the CM2. Unfortunately many sequential algorithms converted in such a straightforward, mechanical way are not efficient for a parallel machine. Figure 2 illustrates the point for matrix multiplication [21]. For this reason, much of the research to date in algorithms for parallel machines has been a complete rethinking that has produced some unexpectedly new designs that do not resemble their counterparts for sequential computers $[16,17,21,22]$. 


\section{Dataflow Computers}

Dataflow computers are the most practical form of MIMD fine-grained parallel computers known. They limit the cost of synchronization and afford a high degree of parallelism by replacing control flow with data flow. Under control flow, each processor has an instruction pointers that designates which instructions are enabled for execution. Under dataflow, instructions become enabled for execution by the arrival of required operands.

A dataflow program consists of a set of instruction packets stored in the memory of the dataflow computer. An instruction packet is disabled until all its required operands have arrived. Enabled instruction packets are sent via a distribution network to an array of processors, where they are executed and their results distributed back to instruction packets that await them. If a large number of instruction packets are enabled, a dataflow computer with a large number of processors achieves high parallelism and high utilization. A dataflow computer can offer fine grain parallelism because it can exploit parallelism at the level of individual functions, expressions, and subexpressions [23].

Program statements that operate on arrays of data will achieve high speedups on a dataflow machine. Consider again the earlier example of a computation over a grid of points $v(i, j)$. In a dataflow computer, all the assignment instructions for all the grid

points would be enabled in parallel; each would await four operands generated by its four neighbors, and would then produce a new result. The speed of the machine would be directly proportional to $N / M$ for $N$ grid points and $M$ processors. These findings were confirmed by a study we performed jointly with NASA and DARPA in 1984 [24]. Benchmark studies on a prototype dataflow computer at the University of Manchester 
have indicated that many sequential programs can also keep all the processors of a dataflow computer busy [25].

A dataflow computer automatically solves the problem of assigning virtual processors (here, instruction packets) to the real processors of the machine: as soon as a virtual processor is enabled by the arrival of needed operands, it is sent to a real processor for execution. Although the ratio of computation to communication time per virtual processor may be low (0.01 to 0.1$)$, utilization of the machine can nevertheless by close to 1 if the program has sufficiently many instructions enabled at the same time.

Aside from a few university and commercial prototypes, no serious commercial dataflow machine is available. There are several reasons for this. The SIMD machine is simpler to build and can be programmed within familiar language concepts; the dataflow machine requires new languages and new compiling technologies based on unfamiliar concepts [19]. The SIMD machine uses a hypercube interconnection network, which is cheap to build; the dataflow machine depends on a high-speed packet switched network, a technology that is only now becoming inexpensive.

Some researchers are studying dataflow languages as source languages for SIMD architectures. Experience with these languages will benefit the programming of all parallel machines. 


\section{Conclusions}

The sequential computer has been the dominant paradigm since the first ENIAC was brought on-line in 1946. We are fast approaching the physical limits of this technology while our computational needs continue to grow. After two decades of experimentation, successful computers containing thousands of processors operating in parallel have been built and are for sale in the market, and early experience with these machines in practice has been highly encouraging. Many challenges lie ahead in computer architecture, algorithms, programming languages, compilers, operating systems, performance evaluation, software engineering, and the vast number of applications.

The new breed of massively parallel machines will in the long run have an impact as profound as microcomputers. These machines are forcing us to rethink our approaches to algorithms: any technology that brings about a change in the manner of organizing work will have far-reaching effects. 


\section{References and Notes}

1. J. J. Dongarra, "Performance of various computers using standard linear equations software in a Fortran environment," Computer Architecture News, 16, 47 (1988).

2. G. Bell, "The future of high performance computers in science and engineering," Comm. ACM, 32, 1091 (September 1989).

3. A. H. Karp and H. P. Flatt, "Measuring Parallel Processor Performance," Comm. $A C M, 33,539$ (May 1990).

4. J. Gustafson, G. Montry, and R. Benner, 'Development of parallel methods for a 1024-processor hypercube,"' SIAM J. Sci. Stat. Comp., 9, 1 (1988).

5. G. C. Fox, "What Have We Learnt from Using Real Parallel Machines to Solve Real Problems?" Hypercube Concurrent Computers and Applications (ACM Press, New York, 1988), pp. 897-955.

6. M. J. Flynn, "Some computer organizations and their effectiveness," IEEE Trans. on Comp. C-21, 948 (September 1972).

7. H. J. Siegel, Interconnection Networks for Large-Scale Parallel Processing: Theory and Case Studies (Lexington Books, 1985).

8. P. J. Denning, “Multigrids and hypercubes," American Scientist, 75, 234 (May 1987).

9. F. Baskett and A. J. Smith, "Interference in Multiprocessor Computer Systems with Interleaved Memory," Comm. of ACM, 19 (June 1976).

10. P. J. Denning, "Is Random Access Memory Random?" American Scientist, 74, 126 (March-April 1986). 
11. R. D. Rettberg, W. R. Crowther, P. P. Carey, and R. S. Tomlinson, "The Monarch Parallel Processor Hardware Design," IEEE Computer, 23, 18-30 (April 1990).

12. D. Hillis, The Connection Machine (MIT Press, Cambridge, MA, 1985).

13. J, R. Fischer, Ed., Frontiers of massively parallel scientific computation. Proceedings of symposium at NASA Goddard Space Flight Center, 24-25 September 1986, NASA Conference Publication 2478.

14. D. Hillis and G. Steele, "Data Parallel Algorithms," Comm. ACM, 12, 1170 (December 1986).

15. J. J. Hopfield and D. W. Tank, "Computing with Neural Circuits, a Model," Science, 233, 625 (1986).

16. S. G. Akl, The Design and Analysis of Parallel Algorithms (Prentice-Hall, Englewood Cliffs, New Jersey, 1989).

17. A. Gibbons and W. Rytter, Efficient Parallel Algorithms (Cambridge University Press, 1988).

18. Inmos, Ltd., Occam Programming Manual (Prentice-Hall, Englewood Cliffs, NJ, 1984).

19. J. R. McGraw, "The VAL Language: Description and Analysis," ACM Trans. on Programming Languages and Systems, 4, 44 (January 1982).

20. J. Backus, "Can Programming be Liberated from the von Neumann Style?" Comm. $A C M, 21,613-641$ (August 1978).

21. W. F. Tichy, "Parallel Matrix Multiplication on the Connection Machine," Int'lJ. High Speed Computing, 2 (January 1989). 
22. H. D. Simon, Ed., Scientific Applications of the Connection Machine (World Scientific Publishing Co., 1989).

23. K. Hwang and F. A. Briggs, Computer Architecture and Parallel Processing (McGraw-Hill, New York, 1984), pp. 748-768.

24. G. B. Adams III, R. L. Brown, P. J. Denning. "An Evaluation Study of Dataflow Computation." Technical Report TR-85.2 (Research Institute for Advanced Computer Science, NASA Ames Research Center, 230-5, Moffett Field, CA 94025, 1985) In this study, seven NASA teams programmed the MIT static dataflow machine with kernel problems from their scientific domains. The machine was still in design and not implemented. Five of the seven teams concluded they could keep all 256 processors busy and achieve the full 1.28 gflops offered by the design. The two others concluded that a scaled-down version of the machine (16 processors) would be adequate. Weak aspects of the design were uncovered and slated for improvement.

25. J. R. Gurd, C. C. Kirkham, and I. Watson, "The Manchester prototype dataflow machine," Comm. ACM, 28, 34 (January 1985). 


\section{Figure Captions}

FIGURE 1. The figures shows four computer architectures for parallel computation. The first three are in common use and the fourth may become common by the end of the decade. Organization (a) is an SIMD machine in which a single control processor broadcasts a stream of instructions to all data processors. Each instruction calls for an operation on data in local memory or for an exchange of data over the interconnection network. Organization (b) is an MIMD machine in which processors running distinct programs carry out operations on data in a set of memories shared by them all. All processor-memory traffic passes through a high-speed interconnection network. Organization (c) is an MIMD machine in which processors use local memory for most operations and occasionally exchange data over an interconnection network.

Organization (d) is an MIMD dfataflow machine in which instruction packets in memory flow to the processors for execution when all their operands are present; processors send results back, where their arrivals trigger new instructions for execution.

FIGURE 2. Experiments on a Connection Machine reveal big differences in performance and illustrate tradeoffs between storage requirements and running times on parallel machines. The graph shows running time for three algorithms for multiplying $N \times N$ matrices on a $\mathrm{CM} 2$ with $2^{15}$ processors. Each curve is labeled with its asymptotic growth rate in $N$. The upper curve is for the standard sequential algorithm that takes time proportional to $N^{3}$ using one processor. The middle curve is an algorithm that uses $N^{3}$ processors and takes time proportional to $\log N$. The lower curve is an algorithm that computes each of the $N^{2}$ results on a separate processor, it takes time proportional to $N$. 
The discontinuities in the two lower curves result from the simulation of virtual processors. For each value of $N=32,40,50,64,80,100,128, \ldots$ in the middle curve the next stage of virtual processor simulation starts, with respectively $2,4,8,16,32,64,128$, ... virtual processors per real processor; at each step the running time doubles and at $\mathrm{N}=256$ the machine runs completely out of memory. When $N>181$ in the lower algorithm, $N^{2}>2^{15}$, and the running time doubles for the same reason. 


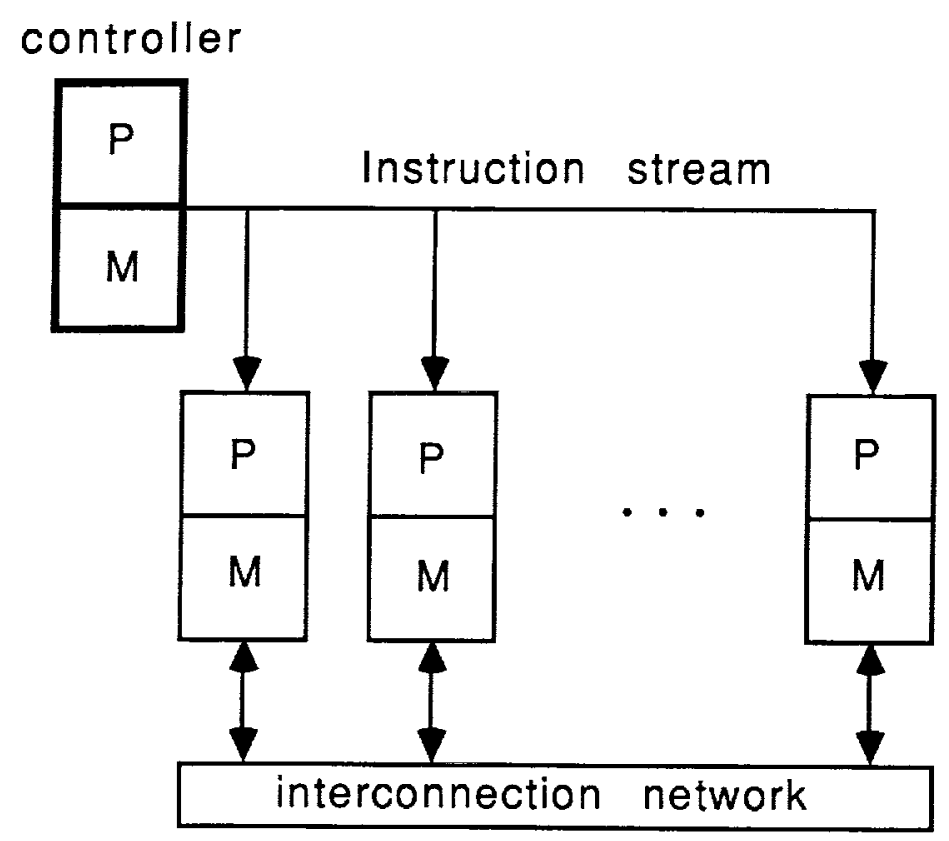

(a) SIMD distributed

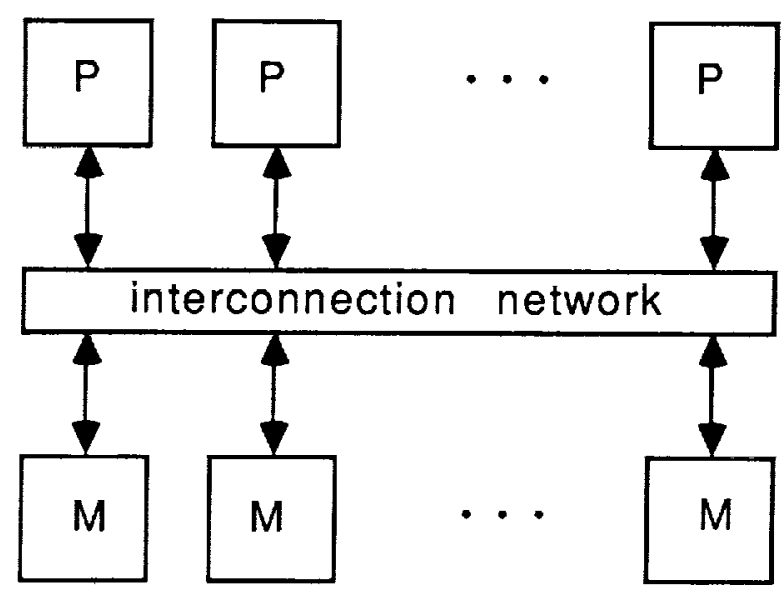

(b) MIMD shared 


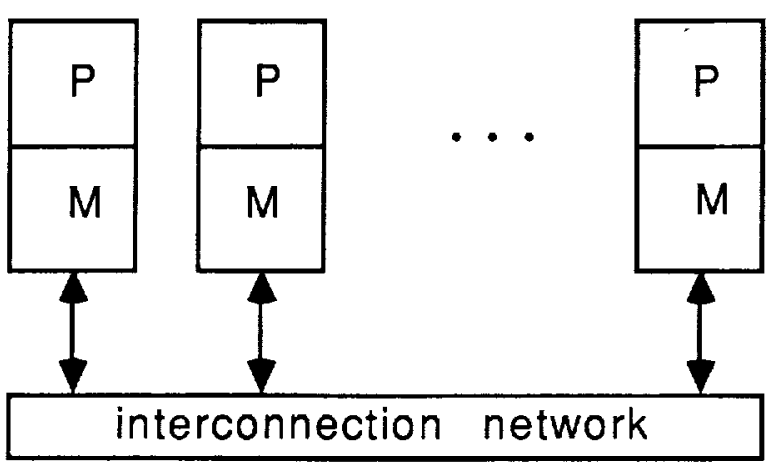

(c) MIMD distributed

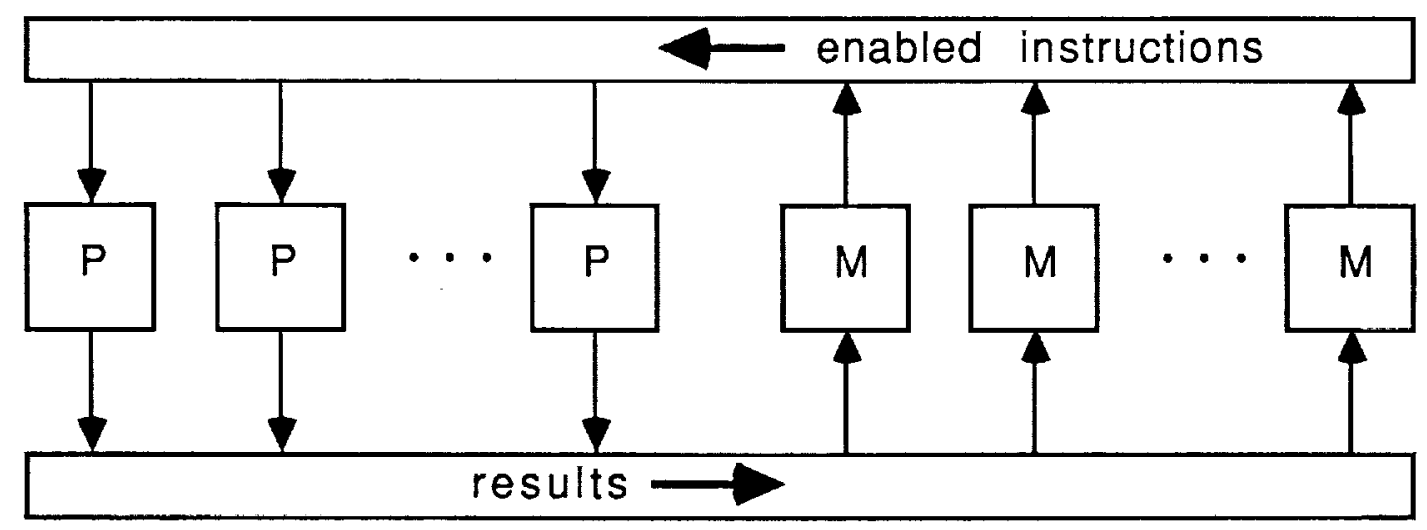

(d) MIMD dataflow 


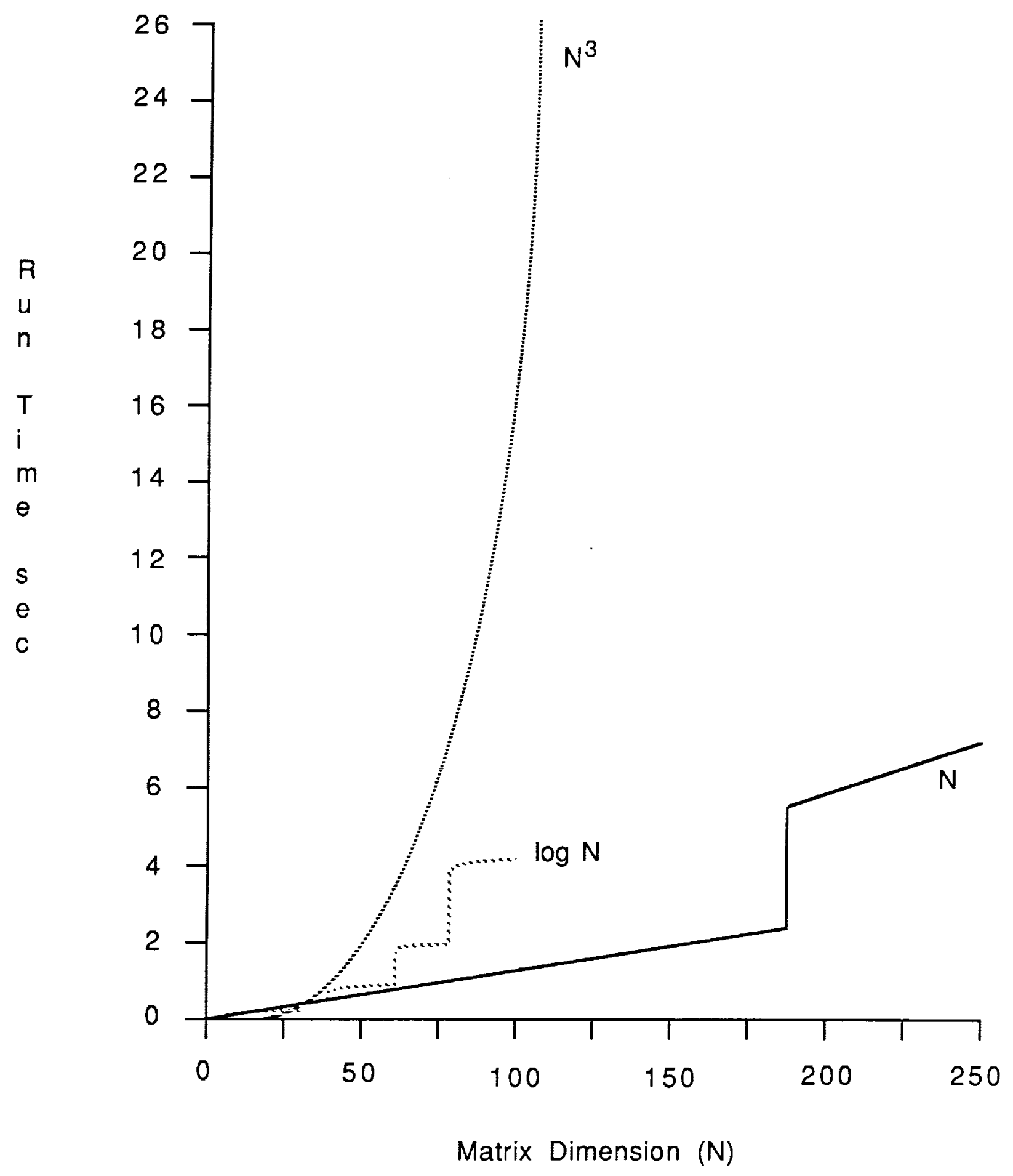

